\title{
A deep VLA map of the gamma-ray binary LS I +61 303 and its environment
}

\author{
Alvaro J. Muñoz-Arjonilla* ${ }^{\dagger}$ \\ Universidad de Jaén \\ E-mail: ajmunoz@ujaen.es
}

\author{
J. Martí, P. Luque-Escamilla, J. A. Combi, and J. R. Sánchez-Sutil \\ Universidad de Jaén \\ E-mail: jmarti@ujaen.es ; peter@ujaen.es ; jcombi@ujaen.es ; \\ jrssutilehotmail.com
}

\author{
V. Zabalza, J. M. Paredes \\ Universitat de Barcelona \\ E-mail: vzabalza@am.ub.es ; jmparedes@ub.edu
}

\begin{abstract}
LS I +61 303 is a gamma-ray binary first detected in 1977 (Gregory \& Taylor 1978). It has been studied for last three decades by many authors, with very interesting properties of this system being revealed, ranging from the historically observed $26.5 \mathrm{~d}$ periodic radio outbursts of nonthermal nature (Taylor \& Gregory 1982) to the recent detection of variable TeV emission with the MAGIC Cherenkov telescope (Albert et al. 2006). There are two main competing models to explain the spectral energy distribution observed in gamma-ray binaries: a microquasar X-ray binary and a non-accreting pulsar interacting with the envelope of the rapidly rotating Be star. In this work, our original motivation was to search for very extended radio features of any kind associated to this system which could help to solve microquasar / pulsar debate.

We present a new radio map of LS I +61 303 obtained after combining VLA archive data at $6 \mathrm{~cm}$ and covering a field of view of about 12 arc-minute, which probably is the deepest centimetric radio image of this system reported up to date at this wavelength (rms noise about $13 \mu \mathrm{Jy} \mathrm{beam}^{-1}$ ). It shows no extended radio features which could be associated to LS I +61 303 at this level of sensitivity. This fact allows us to establish upper limits to the energy content of any possible faint bipolar radio lobes at parsec scales in case they existed. We also report a list of the compact radio sources in the field, some of them not catalogued before, as well as an improved radio image of the planetary nebula WeBo1 (Bond et al. 2003) also observed in this field.
\end{abstract}

From Planets to Dark Energy: the Modern Radio Universe

October 1-5 2007

The University of Manchester, UK

\footnotetext{
* Speaker.

${ }^{\dagger}$ The authors acknowledge support by grants AYA2004-07171-C02-02 and AYA2004-07171-C02-01 from the Spanish government, FEDER funds. This has been also supported by Plan Andaluz de Investigación of Junta de Andalucía as research group FQM322.
} 


\section{A search of possible extended radio features associated to LS I +61 303}

Since its discovery, LS I +61 303 was suspected to be associated COS-B gamma-ray source 2CG $135+01$. Today the system is considered to be the true counterpart candidate to the unidentified high energy $(\geq 100 \mathrm{MeV})$ source 3EG J0241+6103, specially after LS I +61 303 was also detected as a variable $\mathrm{TeV}$ source with the MAGIC Cherenkov telescope (Albert et al. 2006). It is at present one of the few members of the selected class of gamma-ray binaries, with the others being the systems Cygnus X-1, PSR B1259-63 and LS 5039. The physical scenario behind the radio and gamma-ray emission is yet a matter of debate. Two different models are currently being considered in order to explain the full spectral energy distribution: a microquasar X-ray binary (Bosch-Ramon et al. 2006) and a non-accreting pulsar interacting with the envelope of the rapidly rotating Be star (Dubus 2006). Both observational and theoretical work has been reported in support of both models in different members of the gamma-ray binary group. Although the pulsar scenario tends to be recently preferred based on VLBI observations (Dhawan et al. 2006), the situation is not yet fully understood (Romero et al. 2007).

In an attempt to help to discriminate between the two proposed models, we searched for extended radio features at parsec scales. For instance, the detection of faint bipolar radio lobes could be regarded as evidence for a large scale collimated outflow and hence support a microquasar scenario. We retrieved archive data from VLA projects UT0002 and GM0014 carried out in 1992 and 1993 in the CnD hybrid configuration of the array. This was the best available choice to ensure sensitivity to extended emission at arc-minute scales, equivalent to $\sim 1 \mathrm{pc}$ at the LS I +61 303 distance. After concatenating these VLA runs, the resulting map (Fig. 1) significantly improves by $\mathrm{a} \sim \sqrt{2}$ factor the rms noise obtained by Martí et al. (1998) in a previous similar work.

\section{Energy considerations for possible radio lobes}

Despite our non-detection of arc-minute extended radio emission associated to LS I+61303, our data enable us to put upper limits on the total energy content of any possible radio lobe or other extended feature as a function of its size. Reasonable values for the dimensions of large scale lobes would be in the 0.1 to few pc range, as often observed in galactic microquasars. Assuming equipartition conditions, the allowed region in the Energy-Size plane can be constrained. Details are given in Muñoz-Arjonilla et al. (in preparation).

\section{Radio sources detected in the vicinity of LS I +61 303}

A total of 19 compact radio sources are detected in the vicinity of LS I +61 303 with flux density above the $4 \sigma$ level. Ten of them are new ones respect to those listed in the previous work by Martí et al. (1998). The planetary nebula WeBol (Bond et al. 2003) is also well detected in this very deep image. 


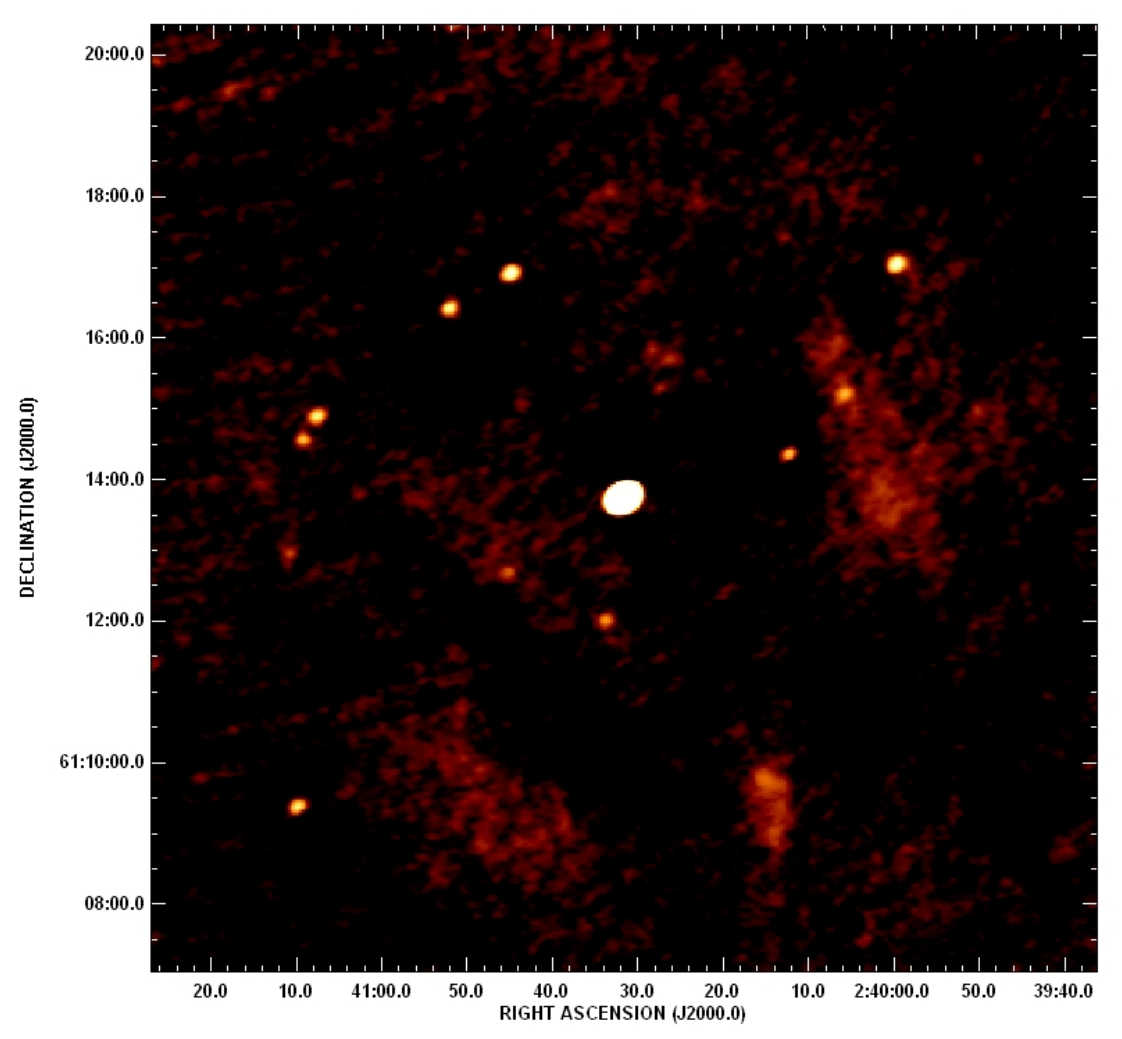

Figure 1: Final VLA map centered on the LS I +61 303 position showing different faint compact and extended radio sources in the field. Phase self calibration and natural weight were used. Artifacts due to the hourly variability of our target have been minimized by subtracting the central variable point source with a custom AIPS task written by us. A constant point source with the average flux density was later artificially added for cosmetic purposes. The synthesized beam is practically a 11 arc-second circular Gaussian. The achieved rms noise is about $13 \mu \mathrm{Jy}_{\text {beam }^{-1}}$.

\section{References}

[1] Albert, J., et al., 2006, Science, 312, 1771

[2] Bond, H.E., et al., 2003, AJ, 125, 260

[3] Bosch-Ramon, V., et al., 2006, A\&A, 459, L25

[4] Dhawan, V., Mioduszewski, A., Rupen, M., 2006, PoS, Proc. VI Microquasar Workshop, p. 52.1

[5] Dubus, G., 2006, A\&A, 456, 801

[6] Gregory, P. C., Taylor, A. R., 1978, ApJ, Nature, 272, 704

[7] Heinz, S., 2002, A\&A, 388, L40

[8] Martí, J., Peracaula, M., Paredes, J. M., et al., 1998, A\&A, 329, 951

[9] Romero, G., et al., 2007, A\&A, arXiv:0706.1320v1 astro-ph

[10] Taylor, A. R., Gregory, P. C., 1982, ApJ, 255, 210 